\title{
Targeting De Novo Lipogenesis and Cholesterol Biosynthesis Simultaneously is a Novel Therapeutic Option for Hepatocellular Carcinoma
}

\author{
This article was published in the following Dove Press journal:
} Journal of Hepatocellular Carcinoma

\author{
Qian Liu' \\ Xifeng Dong ${ }^{2}$ \\ 'Tianjin Lung Cancer Institute, Tianjin \\ Medical University General Hospital, \\ Tianjin 300020, People's Republic of \\ China; ${ }^{2}$ Department of Hematology, \\ Tianjin Medical University General \\ Hospital, Tianjin 300020, People's \\ Republic of China
}

\begin{abstract}
Hepatocellular carcinoma (HCC) is one of the most common and serious types of cancer in the world. Currently, the treatment options for patients with HCC are limited. Lipid metabolic alterations are being recognized as a therapeutic target in the past few years. De novo lipogenesis has been frequently observed in HCC. Fatty acid synthase (FASN) is the key enzyme of de novo lipogenesis. Previous studies have indicated that loss of FASN suppresses the growth of HCC cells, but it cannot completely prevent HCC formation in vivo. Thus, other mechanisms that can support HCC tumor formation in the absence of de novo lipogenesis maybe existed. In a study recently published in Gut, Che and colleague investigated the functions of Fasn in HCC mouse model and explore the crosstalk between de novo lipogenesis and cholesterol biosynthesis-associated pathway during HCC development. These findings highlight the simultaneous inhibition of de novo lipogenesis and cholesterol biosynthesis as a novel therapeutic and prevention strategy for HCC.
\end{abstract}

Keywords: hepatocellular carcinoma, HCC, cholesterol, fatty acid, lipogenesis, biosynthesis

Hepatocellular carcinoma (HCC) is the fourth commonest diagnosed cancers in the world. ${ }^{1}$ It is one of the few cancers in China with incidence rates that continue to rise in recent years. ${ }^{2}$ For patients with HCC, the treatment options are limited and vary significantly by disease extent. Multikinase inhibitors, such as sorafenib and regorafenib, have always been the first-line treatment regime of advanced HCC, but their long-term survival benefit is limited. ${ }^{3}$ Therefore, exploring disease mechanisms and finding new therapeutic targets of HCC could possibly extend the life of patients more effectively.

A growing body of evidence suggests that cancer cells need to reprogram the metabolism in order to support the increased biomass synthesis for their survival and growth. ${ }^{4}$ Metabolic reprogramming in cancer cells includes the upregulation of glycolysis, glutaminolysis, lipid metabolism, pentose phosphate pathway, and other biosynthetic pathways. ${ }^{5}$ Among them, lipid metabolic alterations are increasingly being recognized in the past few years. ${ }^{6}$ Increased de novo lipogenesis (synthesis of fatty acids from acetyl-CoA) has been observed in various cancer types, including HCC. ${ }^{7,8}$ Fatty acid synthase (FASN) is the key enzyme of de novo lipogenesis. Previous findings have indicated that HCC tissues have higher FASN expression levels as compared with non-tumorous liver tissues. ${ }^{9,10}$ Additionally, loss of FASN function in combination with c-Met expression has been shown to suppress the tumorigenesis in the livers of mice with activated protein kinase B (PKB)
Correspondence: Xifeng Dong Department of Hematology, Tianjin Medical University General Hospital, Tianjin 300020, People's Republic of China

Email dongxifeng@sdu.edu.cn
Journal of Hepatocellular Carcinoma 2021:8 19-21

19

(c) (i) (5) 2021 Liu and Dong. This work is published and licensed by Dove Medical Press Limited. The full terms of this license are available at https://www.dovepress.com/terms. work you hereby accept the Terms. Niporate the Creative Commons Attribution - Non Commercial (unported, v3.0) License (http:///creativecommons.org/licenses/by-nc/3.0/). By accessing the permission for commercial use of this work, please see paragraphs 4.2 and 5 of our Terms (https://www.dovepress.com/terms.php). 
overexpression. ${ }^{11}$ However, FASN silencing only suppressed the growth of HCC cells, but it could not completely prevent HCC formation in vivo. ${ }^{12}$ These emerging observations suggest that other mechanisms that can support HCC tumor formation in the absence of de novo lipogenesis maybe existed. Nowadays, cholesterol biosynthesis alteration is recognized as one of the cancer features. $^{13}$ The rate-limiting enzyme of cholesterol biosynthesis, $\beta$-hydroxy $\beta$-methylglutaryl-CoA reductase (HMGCR), is frequently upregulated in HCC tissues. ${ }^{10}$ Moreover, drugs targeted HMGCR, such as statins, have been demonstrated to reduce the risk of HCC development in a large-scale epidemiological study. ${ }^{14}$ All these evidence suggest that the cholesterol biosynthesis pathway plays a critical role in HCC initiation and progression. In a study recently published in Gut, titled "Cholesterol biosynthesis supports the growth of hepatocarcinoma lesions depleted of fatty acid synthase in mice and humans", Che et $\mathrm{al}^{15}$ investigated the functions of Fasn in HCC mouse model and explore the crosstalk between de novo lipogenesis and cholesterol biosynthesis-associated pathway during HCC development.

In this study, the authors generated $\mathrm{Fasn}^{L K O}$ mice to explore whether sgPten/c-Met-driven HCC tumorigenesis requires Fasn and Fasn-mediated de novo lipogenesis. The Fasn $^{L K O}$ mouse model for sgPten/c-Met-driven HCC tumorigenesis was generated through two distinct approaches. One approach is to generate $\mathrm{Fasn}^{L K O}$ mice by breeding Fasn ${ }^{f l / f l}$ mice with AlbCre mice and then inject the expression plasmids of sgPten and c-Met into Fasn ${ }^{L K O}$ mice. Another approach is to hydrodynamically inject the expression plasmids of sgPten, c-Met and Cre into Fasn ${ }^{\text {fl/fl }}$ mice. All mice in the control group (sgPten/c-Met-injected $\mathrm{Fash}^{f / f l}$ mice) developed HCC by 8-10 weeks after injection. In contrast, all Fasn ${ }^{L K O}$ mice with sgPten and c-Met expression stayed healthy during this period. However, HCC tumorigenesis was not abolished by Fasn deletion. Within 14-40 weeks after injection, all sgPten/c-Metinjected Fasn ${ }^{L K O}$ mice exhibited malignant lesions in the liver. The authors performed a global gene expression microarray to further elucidate the underlying mechanism of Fasn in HCC tumorigenesis. They found that Fasn deletion leads to Srebp2 activation, which further leads to the increased expression of genes associated with cholesterol biosynthesis.

By comparing the lipid profiles between Fasn positive and negative HCC tissues, the authors found that the levels of triacylglycerols, triacylglycerols, and free fatty acids were lower and cholesterol ester was higher in Fasn negative HCC tissues. Additionally, the analyses of fatty acid composition and source showed that the overall contribution of dietary fatty acid in Fasn negative HCC increased significantly. These observations suggest that when de novo lipogenesis is suppressed, fatty acid uptake is the main compensation mechanism for obtaining the fatty acid required for HCC tumorigenesis. Furthermore, by combining the genomics and lipidomics analysis, the authors found that inhibit de novo lipogenesis may trigger Srebp2 activation and increase cholesterol biosynthesis during HCC tumorigenesis. Therefore, they hydrodynamically injected $\operatorname{sgPten} / \mathrm{c}-\mathrm{Met}$ and dnSrebp2 plasmids into Fasn $^{L K O}$ mice (sgPten/c-Met/dnSrebp2 Fasn ${ }^{L K O}$ mice) to evaluate the effect of Srebp2 on HCC tumorigenesis. All control mice injected with sgPten/c-Met and pT3 empty vector exhibited liver tumors within 15-31 weeks after injection; however, sgPten/c-Met/dnSrebp2 mice did not develop liver tumors until 50 weeks after injection. These findings indicate that the simultaneous inhibition of de novo lipogenesis mediated by Fasn and cholesterol biosynthesis driven by Srebp2 may completely prevent HCC tumorigenesis. Further cell biological experiments conducted on human HCC cell lines showed that silencing FASN by siRNA might lead to the compensatory activation of SREBP2/HMGCR cascade in human HCC. Additionally, the mRNA levels of $S R E B P 2$ and its transcriptional programme-associated genes were upregulated in HCC tissues, as well as the cholesterol pathway activation was a poor prognostic factor in patients with HCC.

From the previous studies, we have already known that abnormal activation of FASN and FASN-mediated de novo lipogenesis are necessary for HCC initiation. Suppressing de novo lipogenesis mediated by FASN can inhibit cancer cell proliferation of $\mathrm{HCC}$ both in vitro and in vivo. However, in the case that the de novo lipogenesis is completely inhibited, HCC can still maintain survival and proliferation through other mechanisms. The present study reported for the first time that Fasn silencing and inhibition of de novo lipogenesis mediated by Fasn delays HCC formation, but does not completely prevent sgPten/c-Metdriven HCC tumorigenesis in mouse models. In addition, the upregulation of cholesterol biosynthesis driven by Srebp2 in the liver of Fasn ${ }^{L K O}$ mice eventually led to the occurrence of HCC. These findings reveal a novel biochemical interaction between de novo lipogenesis and cholesterol biosynthetic pathways in HCC initiation. Blocking the biosynthesis of cholesterol can completely 
prevent the HCC formation in mice when Fasn is lost. All the above findings strongly suggest that simultaneous inhibition of de novo lipogenesis and cholesterol biosynthesis is a novel therapeutic and prevention strategy for HCC. Moreover, this molecular mechanism needs to be further verified in other tumor types as it may occur widely in various cancer types.

\section{Abbreviations}

HCC, hepatocellular carcinoma; FASN, fatty acid synthase; $\mathrm{PKB}$, protein kinase $\mathrm{B}$; HMGCR, $\beta$-hydroxy $\beta$ methylglutaryl-CoA reductase.

\section{Data Sharing Statement}

Not applicable.

\section{Ethics Approval and Consent to Participate}

Not applicable.

\section{Consent for Publication}

Not applicable.

\section{Funding}

There is no funding to report.

\section{Disclosure}

The authors report no conflicts of interest for this work.

\section{References}

1. Siegel RL, Miller KD, Jemal A. Cancer statistics, 2019. CA Cancer J Clin. 2019;69(1):7-34. doi:10.3322/caac.21551
2. Feng RM, Zong YN, Cao SM, Xu RH. Current cancer situation in China: good or bad news from the 2018 Global Cancer Statistics? Cancer Commun. 2019;39(1):22. doi:10.1186/s40880-019-0368-6

3. Kudo M. Systemic therapy for Hepatocellular Carcinoma: 2017 update. Oncology. 2017;93(Suppl 1):135-146. doi:10.1159/000481 244

4. Pavlova NN, Thompson CB. The emerging hallmarks of cancer metabolism. Cell Metab. 2016;23(1):27-47. doi:10.1016/j.cmet.2015. 12.006

5. Wang Y, Xia Y, Lu Z. Metabolic features of cancer cells. Cancer Commun. 2018;38(1):65. doi:10.1186/s40880-018-0335-7

6. Kuo CY, Ann DK. When fats commit crimes: fatty acid metabolism, cancer stemness and therapeutic resistance. Cancer Commun. 2018; 38(1):47. doi:10.1186/s40880-018-0317-9

7. Rohrig F, Schulze A. The multifaceted roles of fatty acid synthesis in cancer. Nat Rev Cancer. 2016;16(11):732-749. doi:10.1038/nrc.20 16.89

8. Lu S, Wang Y. Nonmetabolic functions of metabolic enzymes in cancer development. Cancer Commun. 2018;38(1):63. doi:10.1186/ s40880-018-0336-6

9. Che L, Pilo MG, Cigliano A, et al. Oncogene dependent requirement of fatty acid synthase in hepatocellular carcinoma. Cell Cycle. 2017;16(6):499-507. doi:10.1080/15384101.2017.1282586

10. Calvisi DF, Wang $C$, Ho C, et al. Increased lipogenesis, induced by AKT-mTORC1-RPS6 signaling, promotes development of human hepatocellular carcinoma. Gastroenterology. 2011;140(3):1071-10 83. doi:10.1053/j.gastro.2010.12.006

11. Li L, Pilo GM, Li X, et al. Inactivation of fatty acid synthase impairs hepatocarcinogenesis driven by AKT in mice and humans. J Hepatol. 2016;64(2):333-341. doi:10.1016/j.jhep.2015.10.004

12. Cao D, Song X, Che L, et al. Both de novo synthetized and exogenous fatty acids support the growth of hepatocellular carcinoma cells. Liver Int. 2017;37(1):80-89. doi:10.1111/liv.13183

13. Murai T. Cholesterol lowering: role in cancer prevention and treatment. Biol Chem. 2015;396(1):1-11. doi:10.1515/hsz-2014-0194

14. Singh S, Singh PP, Singh AG, Murad MH, Sanchez W. Statins are associated with a reduced risk of hepatocellular cancer: a systematic review and meta-analysis. Gastroenterology. 2013;144(2):323-332. doi:10.1053/j.gastro.2012.10.005

15. Che L, Chi W, Qiao Y, et al. Cholesterol biosynthesis supports the growth of hepatocarcinoma lesions depleted of fatty acid synthase in mice and humans. Gut. 2020;69(1):177-186. doi:10.1136/gutjnl-20 $18-317581$

\section{Publish your work in this journal}

The Journal of Hepatocellular Carcinoma is an international, peerreviewed, open access journal that offers a platform for the dissemination and study of clinical, translational and basic research findings in this rapidly developing field. Development in areas including, but not limited to, epidemiology, vaccination, hepatitis therapy, pathology and molecular tumor classification and prognostication are all considered for publication. The manuscript management system is completely online and includes a very quick and fair peer-review system, which is all easy to use. Visit http://www.dovepress.com/ testimonials.php to read real quotes from published authors. 